\title{
Acquisition and Quantification of Ion Images with a Camera-Based Detection System and Classical Quantification Algorithms
}

\author{
F. Michiels, W. Vanhoolst, P. Van Espen, and F. Adams \\ Department of Chemistry, University of Antwerp (U.I.A.), Wilrijk, Belgium
}

\begin{abstract}
The quantitative analytical characteristics of a camera-based detection system for secondary ion microscopy are studied in detail. Through multiple exposures, ion images with large dynamic ranges are obtained. The quantification of these images by the sensitivity factor method and the matrix ion species ratio method is described and evaluated by the analysis of steel and aluminum standard reference materials. (J Am Soc Mass Spectrom 1990, 1, 37-52)
\end{abstract}

$\mathrm{E}$ ver since the development of imaging secondary ion mass spectrometry (SIMS) [1], researchers have shown great interest in the quantification of ion images [2-4]. In the last decade, the potentialities of imaging SIMS have grown rapidly. This is due to both the development and commercial availability of cost-effective image processing equipment and advances in the technology of ion microprobe mass spectrometry and ion microscopy.

In the ficld of ion microprobe analysis, much effort was dedicated to the development of brighter ion sources, resulting in narrower beams and thus better lateral resolution. With the state-of-the-art liquid metal ion sources (LMIS), probe sizes down to $50 \mathrm{~nm}$ can now be achieved $[5,6]$.

In ion microscopy, the new generation instruments allow a lateral resolution below $1 \mu \mathrm{m}$ [7], and registration of the images is greatly improved. There are now two main alternatives for image acquisition. The resistive anode encoder (RAE) detector is a position-sensitive pulse-counting device that allows direct imaging of secondary ion currents up to $10^{5}$ counts/s. It provides a lateral resolution of 300 lines, and the quantification is straightforward, as it relies on direct counting of the secondary ions in every pixel. The system is very "transparent" to the user, its main disadvantage being its limited dynamic range [8].

The combination of a single or dual microchannel plate with a sensitive camera is a frequently employed alternative. Various experimental designs are described in the literature [9-12]. This camera-based setup does not suffer from a limitation in the maximum count rate [13], but the quantification of the ion images is more troublesome because it is dependent on the not necessarily linear, stable, or laterally homogeneous response of the image acquisition system.

Address reprint requests to F. Adams, Department of Chemistry, University of Antwerp (U.I.A.), Universiteitsplein 1, B-2610 Wilrijk, Belgitum.
The combination of image processing and ion microscope or ion microprobe analysis allows extension of the common capabilities of SIMS (mass spectrum analysis, depth profiling, etc.) with a number of very interesting new features such as retrospective depth profiling, line analysis, and three-dimensional analysis $[10,14,15]$. Despite this promising evolution, the progress here is hampered by the quantification problems. Commonly used methods, such as the local thermodynamic equilibrium (LTE) model, the sensitivity factor (SF) method [16-18], the matrix ion species ratio (MISR) method [19, 20], and ion implantation [21] have all been applied with varying degrees of success to convert the ion images from intensity space to concentration space $[2,3,9,22-27]$.

In this paper we describe the implementation of a camera-based detection system and image processing facilities in our laboratory. The analytical characteristics of the camera-based system with respect to quantification are evaluated in detail. A methodology for ion image quantification is worked out and applied to steel and aluminum standard reference materials.

\section{Instrumentation}

\section{Description of the Instrumentation}

Figure 1 gives an overview of the instrumentation used for this work. The SIMS projects the ion image on a microchannel plate, which converts the ions into electrons that are projected onto a fluorescent screen 18 $\mathrm{mm}$ in diameter. This screen can be viewed with two cameras. The standard camera (LHésa) has a medium sensitivity $(0.1 \mathrm{~lx})$ and is used for daily tuning and alignment of the apparatus. The second camera is high-sensitivity $\left(10^{-4}\right.$ lx) MTI-66 silicon intensified target (SIT) camera that is used for image acquisition. The SIT camera is coupled to the ion microscope by the use of $50 \mathrm{~mm} f / 1.2$ and $28 \mathrm{~mm} f / 2.0$ Canon lenses, which 


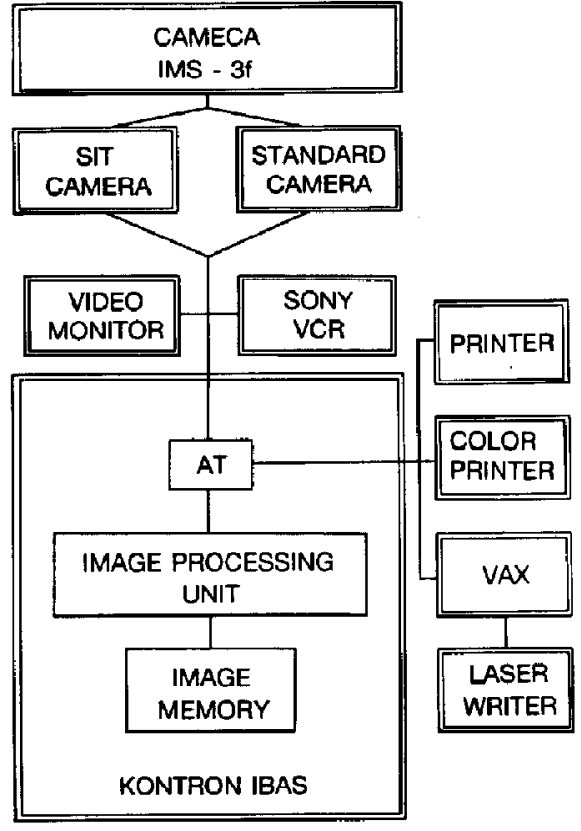

Figure 1. Survey of the instrumentation.

provide optimum light collection from the fluorescent screen and the right magnification to project an image of the fluorescent screen on the faceplate of the camera, thus fully exploiting the lateral resolution of the camera. This camera will be described in more detail later.

The output of the cameras can be directed to a Kontron image processor for online processing, or to a Sony Umatic video cassette recorder (VCR) for image storage. The VCR produces a substantial background and cannot be used for processing images with low intensity.

The Kontron image processing system consists of three main elements. The control processor is an $\mathbf{8 0 2 8 6}$ under MS-DOS (IBM AT). The image processing unit contains a pipeline image processor of $10 \mathrm{Mips}$ (million instructions per second). It is connected to a video memory board (VMB) with 8 Mbytes of RAM. The real-time video (RTV) board in the image processing unit is a 16-bit-deep register and is used for the image processing itself (frame integration, image arithmetic, etc.). The VMB consists of an 8-bit-deep memory plane that can be divided into images of variable pixel size. About seventy $384 \times 256$ images can be stored simultaneously in the VMB.

The software allows a wide variety of image processing functions, from features such as filtering, contour enhancement, frame arithmetic, real-time integration, and phase separation and identification up to twodimensional fast Fourier transform and automatic " $3 \mathrm{D}$ slicing."

Images can be stored on the hard disks of the control units, on floppy disks, or on the tape unit of a
VAX 780 or a $\mu \mathrm{VAX}$, Output can be directed to either a laser writer or a color jet printer. For high-quality reproduction, a high-resolution TV monitor is available.

The software of the SIMS control computer (HP 9836) was adapted to allow automated recording of ion images and measurement of the secondary ion currents. In general, the adaptation of the software for image acquisition is straightforward and can be fitted to the user's needs.

\section{Sensitivity of the Camera-Based Detection System}

One of the major aims of the implementation of the sensitive camera was to extend the detection down to the level of individual secondary ions. The sensitivity and background of the system were evaluated by integrating 20,000 frames (11 min) of an image of $\mathrm{Al}_{4}^{+}$(28 counts/s), as sputtered from an aluminum target covered with a copper grid. With the ion pump at the exit slit of the mass spectrometer turned off [11] and appropriate settings of the camera, secondary ion images without background can be obtained. The sensitivity is probably limited only by the quantum efficiency of the conversion of secondary ions to electrons at the microchannel plate.

\section{Evaluation of the Analytical Characteristics of the SIT Camera}

The camera can be operated in either manual or automatic mode. In automatic mode, the sensitivity is automatically adapted to the illumination conditions by automatic gain, accelerating voltage, and black level control. In manual mode, the operator can independently vary the gain, the accelerating voltage, and the black (pedestal) level of the camera output by means of external controls.

Before attempting any quantitative analysis with the camera, we undertook a systematic study of its quantitative analytical characteristics. For these experiments, we used the camera while viewing test patterns and employed the image processor to measure the output signal. From the experiments, a protocol for camera tuning was developed. The most important conclusions will be presented here.

Figure 2 shows the measured gray value as a function of the light intensity. The data were acquired by recording the image of a homogeneously illuminated white paper. The camera was operated in the manual mode, and the light intensity was varied with a diaphragm. Apart from the deviations due to imperfect settings of the black level, a straight line was obtained for gray values between 0 and 200 . Similar results were obtained for other scene illumination levels and scenes consisting of a white square against a gray background. Overall, the transfer function of the camera was proved to be linear.

Figure 3 shows a similar experiment for the combination of the microchannel plate with the camera. The 


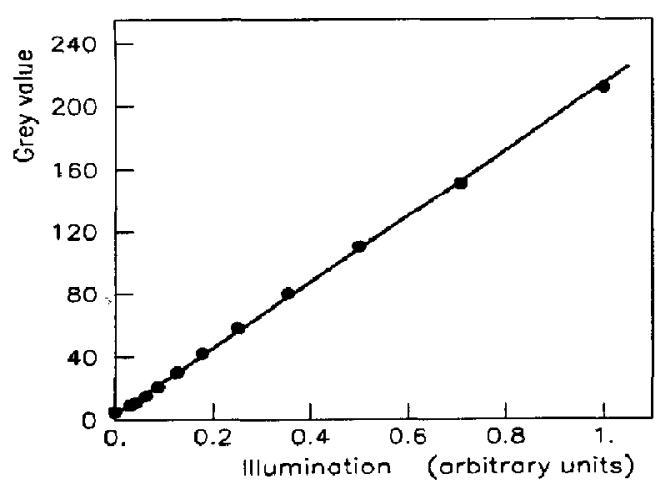

Figure 2. Transfer function of the SIT camera. The measured gray value is plotted versus the light intensity.

secondary ion current, as measured with the electron multiplier detector, is plotted along the $x$ axis. The results indicate that the response of the microchannel plate is linear from 50 to least $5 \times 10^{5}$ counts/s. It is to be stressed that this conclusion does not necessarily hold for other types of cameras because a camera with a lower sensitivity would require a higher amplification at the microchannel plate, and nonlinearities and eventually saturation are expected at high light levels from the microchannel plate-fluorescent screen system. We can conclude that for the system used in this work there is a linear relation between the total secondary ion intensity and the mean gray value as registered with the image processor.

A test pattern consisting of three rectangular areas of different brightness (black, gray, and white) was used to evaluate the influence of incorrect camera tuning on the obtained gray values. This pattern was recorded with different settings of the gain and accelerating voltage of the camera. Because the gain and the accelerating voltage affect the images similarly, one image can be viewed with different conditions of gain and accelerating voltage, each yielding a wellilluminated image. Figure 4 gives the gain-accelerating voltage plane. The bullets correspond to images that

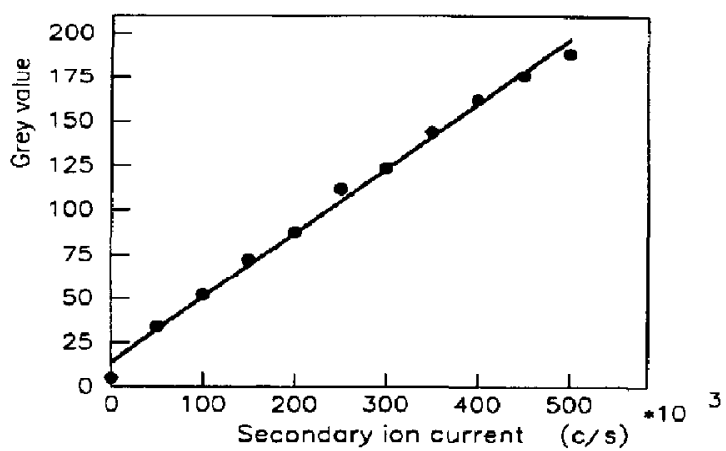

Figure 3. Measured gray value as a function of the secondary ion intensity.

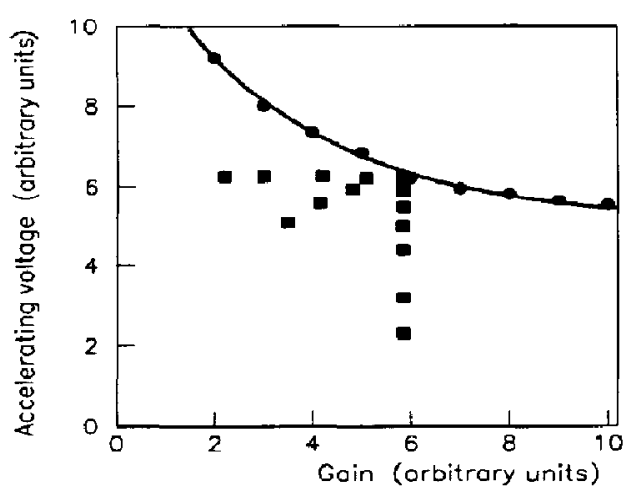

Figure 4. Gain-accelerating voltage plane, indicating the optimum operating conditions of the camera (full line). Beyond this line, the image is overexposed. Experimental points are indicated by bullets and squares for well-illuminated and underexposed images, respectively.

are well illuminated. At these combinations of gain and accelerating voltage, the gray values of the image fully cover the range from 0 to 220 . These points are connected by a full line that indicates the line of optimum camera tuning. Above this line, the camera tube is overexposed. A number of images were intentionally underexposed. These are indicated by the squares in Figure 4. Table 1 summarizes the gray values registered for the three areas in the image. GV1 corresponds to the measured gray value of the black portion, GV2 to the gray portion, and GV3 to the white portion in the image. In analytical practice, the black portion of the image corresponds to the outer edge and the corners of the ion image, where no light is emitted. Table 1 shows that the measured gray-value ratio of the white and gray areas is insensitive to the camera tuning. Moreover, it also demonstrates that the intensity ratios within the image, for example, the ratio GV3/GV2, are undistorted at different levels of gain and accelerating voltage. Similar results were obtained at different scene illumination levels, showing that the gray value ratio is also independent of the light intensity. These two conditions are very important for the cunversion of a gray-value image to a counts per second per pixel image, as will be shown later.

\section{Methodology}

\section{Contrast Types and Artifacts}

The contrast in a recorded secondary ion image is composed of many contrast types, and the task of image quantification can be regarded as the task of separating the concentration contrast of the species of interest from the other contrast sources. Typically four contrast types are discerned.

Topographic contrast occurs when initially present or bombardment-induced roughness of the sample surface shields certain spots of the sample from the pri- 
Table 1. Gray values of a target consisting of a black (GV1), a gray (GV2), and a white (GV3) area, as measured under various conditions of gain, accelerating voltage, and black level

\begin{tabular}{lcccccc}
\hline Gain & $\begin{array}{c}\text { Accelerating } \\
\text { voltage }\end{array}$ & $\begin{array}{c}\text { Black } \\
\text { level }\end{array}$ & GV1 & GV2 & GV3 & GV3/GV2 \\
\hline 10.00 & 5.53 & 8.00 & 3 & 145 & 209 & 1.44 \\
9.00 & 5.65 & 7.95 & 3 & 149 & 214 & 1.44 \\
8.00 & 5.80 & 7.82 & 3 & 150 & 216 & 1.44 \\
7.00 & 5.92 & 7.76 & 3 & 145 & 209 & 1.44 \\
6.00 & 6.22 & 7.65 & 4 & 146 & 210 & 1.44 \\
5.00 & 6.84 & 7.56 & 2 & 142 & 210 & 1.48 \\
4.00 & 7.34 & 7.57 & 2 & 140 & 207 & 1.48 \\
3.00 & 8.00 & 7.60 & 2 & 140 & 209 & 1.49 \\
2.00 & 9.21 & 7.65 & 1 & 138 & 207 & 1.50 \\
5.85 & 6.25 & 7.54 & 2 & 141 & 203 & 1.44 \\
5.85 & 6.08 & 7.54 & 1 & 123 & 180 & 1.46 \\
5.85 & 5.87 & 7.64 & 1 & 108 & 158 & 1.46 \\
5.85 & 5.48 & 7.82 & 2 & 81 & 118 & 1.46 \\
5.85 & 5.00 & 7.90 & 2 & 64 & 96 & 1.50 \\
5.85 & 4.40 & 7.96 & 2 & 54 & 82 & 1.52 \\
5.85 & 3.20 & 8.00 & 2 & 36 & 56 & 1.55 \\
5.85 & 2.30 & 8.10 & 3 & 25 & 38 & 1.52 \\
5.85 & 6.25 & 7.60 & 5 & 146 & 209 & 1.43 \\
4.20 & 6.25 & 7.78 & 1 & 82 & 123 & 1.50 \\
3.00 & 6.25 & 8.05 & 1 & 60 & 93 & 1.55 \\
2.20 & 6.25 & 8.20 & 1 & 45 & 71 & 1.48 \\
5.10 & 6.20 & 7.70 & 2 & 105 & 155 & 1.48 \\
4.82 & 5.95 & 7.78 & 1 & 78 & 118 & 1.51 \\
4.15 & 5.57 & 8.02 & 2 & 50 & 77 & 1.54 \\
3.50 & 5.08 & 8.15 & 1 & 30 & 48 & 1.60 \\
\hline
\end{tabular}

mary beam. Moreover, the angle of incidence varies locally, and this influences the local sputter yield. The contribution of initially present roughness can be minimized by carefully polishing the sample. The influence of the local variations in sputter yield can be eliminated by ratioing the obtained image with a matrix ion image, and for most analyses the contribution of topographical contrast is minor.

Energy contrast is a result of local differences in energy distribution of secondary ions. Due to the limited energy bandpass of the instrument, differences in energy distribution of ions may induce contrast. This effect may be very important in the case of charging samples, where local variations in the amount of charging causes ions to have locally different energy distributions.

Another well-known contrast type is crystallographic contrast. Different microcrystals of a polycrystalline sample have different orientations with respect to the primary beam and may therefore have different sputtering yields. Crystallographic contrast is usually very easy to identify because it is very often possible to recognize the boundaries of individual microcrystals in the image. In this study, the influence of crystallographic contrast is minimized by taking the ratio of the images to a matrix ion image. Moreover, the use of oxygen bombardment usually diminishes the influence of crystallographic contrast because an amorphous oxide layer is formed on top of the sample surface.
A different composition of the sample at different spots will result in concentration contrast. Two major sources for concentration contrast are discerned. Internal concentration contrast is due to local variations solely in the concentration of the imaged species and reflects the real elemental distribution in the sample. Extemal concentration contrast originates from local matrix effects caused by local variations of the matrix composition. These local variations may be due to real differences in matrix composition, such as in the case of inclusions, but may also result from local differences, such as in the case of differential oxygen adsorption from the residual gas or local variations in the concentration of the implanted primary species. Just as in classical SIMS, these matrix effects may cause dramatic changes in the local intensity of the species recorded.

Apart from the contrast sources described above, there are a number of instrumental artifacts that can distort the recorded image.

An ideal image registration instrument could be described by the function

$$
\mathrm{GV}(x, y)=b \cdot I(x, y)
$$

where $\operatorname{GV}(x, y)$ is the gray value at the pixel with coordinates $(x, y), I(x, y)$ is the local secondary ion intensity, and $b$ is a proportionality constant. This assumes that there is no background, that there are no local 


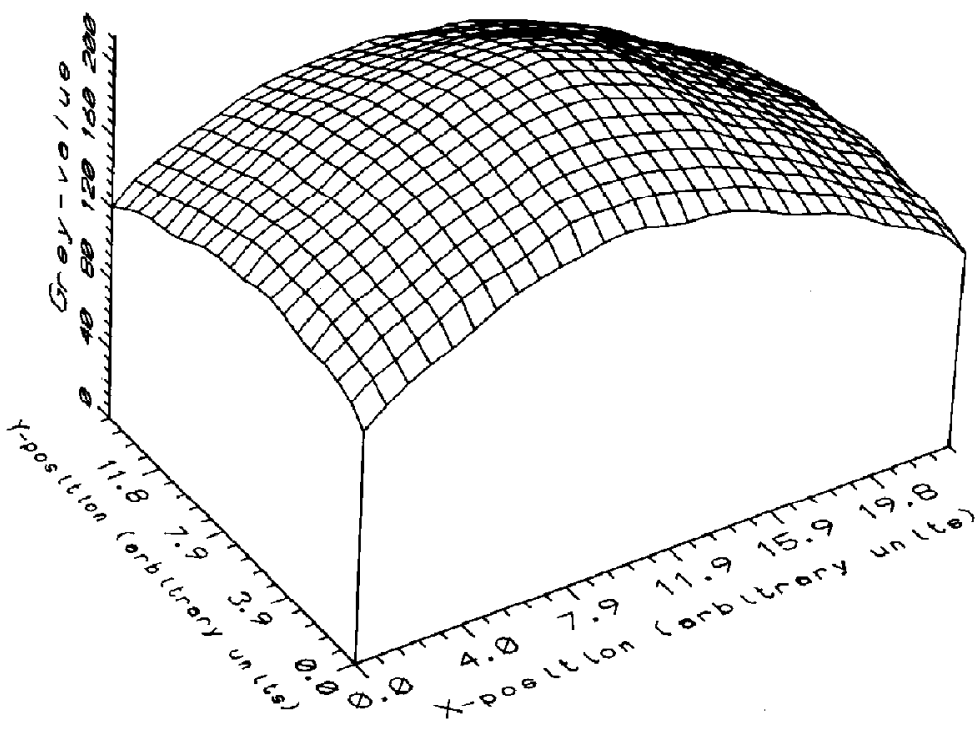

Figure 5. Pseudo-three-dimensional plot of the sensitivity of the camera as a function of position along the $x$ and $y$ axes. This shading plane was recorded as a homogeneously illuminated target was viewed. differences in sensitivity of the recording device, that it is perfectly linear over the whole range of possible intensity values, and that the gray value registered at some pixel is independent of the intensity at other pixels (i.e., that there is no cross-talk).

However, the microchannel plate and the camera show large local differences in sensitivity. The sensitivity of the microchannel plate decreases in the center of the image owing to the degradation caused by the continuous bombardment with secondary ions. The camera has a decreased sensitivity at the edges of the image. Moreover, it is also observed that there is a background signal that differs in intensity from pixel to pixel. Therefore, eq 1 must be modified to

$$
\mathrm{GV}(x, y)=a(x, y)+b(x, y) I(x, y)
$$

The additive part $a(x, y)$ is called the background, whereas the multiplicative part $b(x, y)$ is referred to as shading. Figure 5 shows the shading of the SIT camera as a pseudo-three-dimensional plot.

Equation 2 implies that the gray value at a pixel is independent of the intensity at neighboring pixels, but this proves not to be the case in practice, because cross-talk between neighboring pixels may occur. Two important examples of this were observed in our experiments.

The first example is generally known as the blooming of intense spots. The signal from a very intense spot spreads isotropically to neighboring pixels, increasing the apparent size of the spot. It is assumed that this occurs mainly at the microchannel plate and to a smaller extent in the amplification stage of the SIT camera. The importance of this artifact can be diminished effectively by working at an increased magnification of the projection lenses to increase the size of the features of interest within the image and thus re- duce the relative importance of the blooming, or by reducing the amplification of the microchannel plate.

Another example of cross-talk becomes apparent during work with images with large dynamic ranges. Pixels to the right of very intense spots have a lower sensitivity. Intense spots are therefore followed by darker tails, which may extend over an entire image. Examples of this will be discussed later.

\section{Extended Dynamic Range Imaging}

One of the major limitations of the use of a camera combined with an 8-bit-deep image digitizer is the limited dynamic range within the image. This is especially adverse in the case of SIMS, where large intensity differences within an image are frequently encountered. By integration, however, it is possible to extend the dynamic range appreciably because the image acquisition system has a low background.

Integration is normally performed in real time in the 16-bit-deep RTV memory, which has the capacity to add $256\left(2^{8}\right)$ frames, which each have 8-bit-deep gray values. The addition of 256 frames, called an integration cycle, takes about $8.5 \mathrm{~s}$. After one integration cycle, the 8 most significant bits in the RTV memory can be stored in an 8-bit-deep image memory. The low byte (the 8 least significant bits) often contains noisy information and is discarded. If more integration cycles are added, the image information shifts gradually from the least significant to the most significant bits, and if the maximum capacity is reached $\left(2^{16}-1\right)$, the counter for the specific pixel is reset to zero (black), after which it starts counting up again.

Figure 6a-e shows a 45-ppm manganese image in brass recorded under $1-\mu \mathrm{A} \mathrm{O}_{2}^{+}$bombardment with $6.7 \times 10^{-4} \mathrm{~Pa}$ oxygen backfill, corresponding to a secondary ion current of 4800 counts/s. Figure 6 a shows 


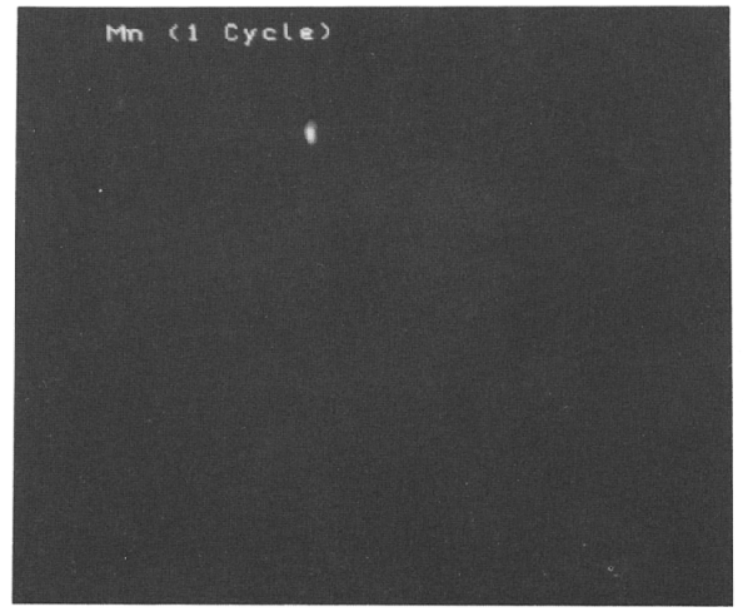

A

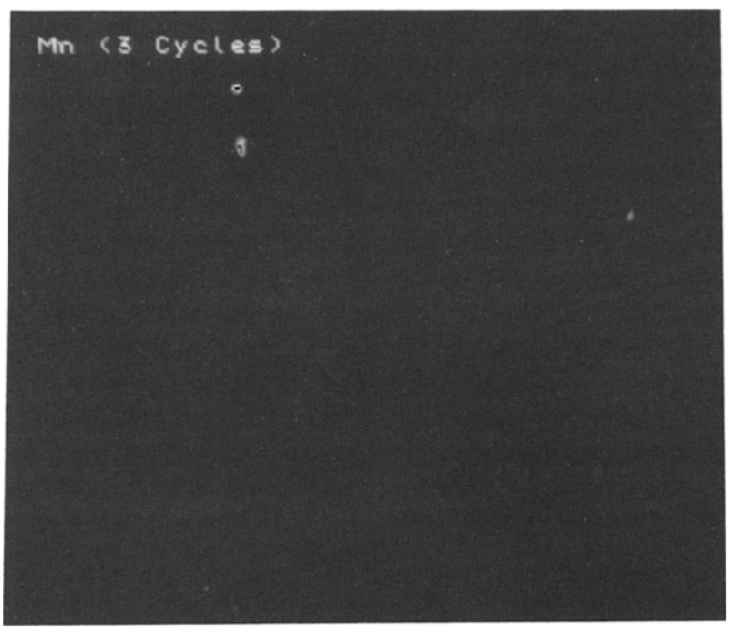

B

Mn (9 Cycles)

Q

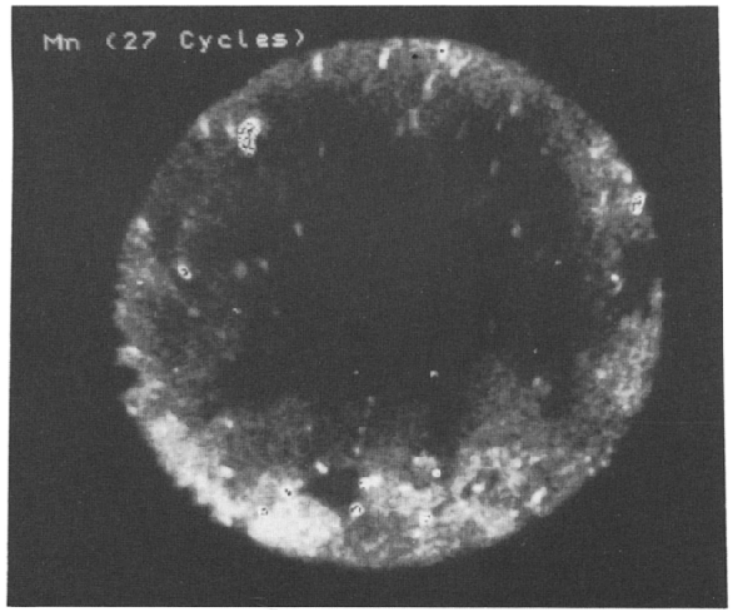

D

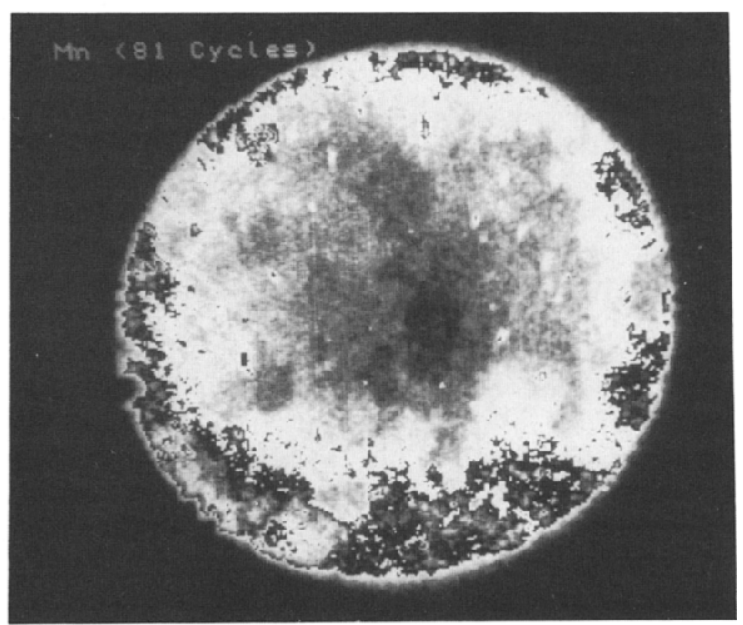

$\mathbf{E}$

Figure 6. Image of $\mathrm{Mn}^{+}$in brass. Integration time: (a) 9, (b) 26, (c) 77, (d) 230, and (e) $691 \mathrm{~s}$. As the exposure time increases, information becomes available on the less intense parts of the image. The diameter of the imaged area is $150 \mu \mathrm{m}$. 


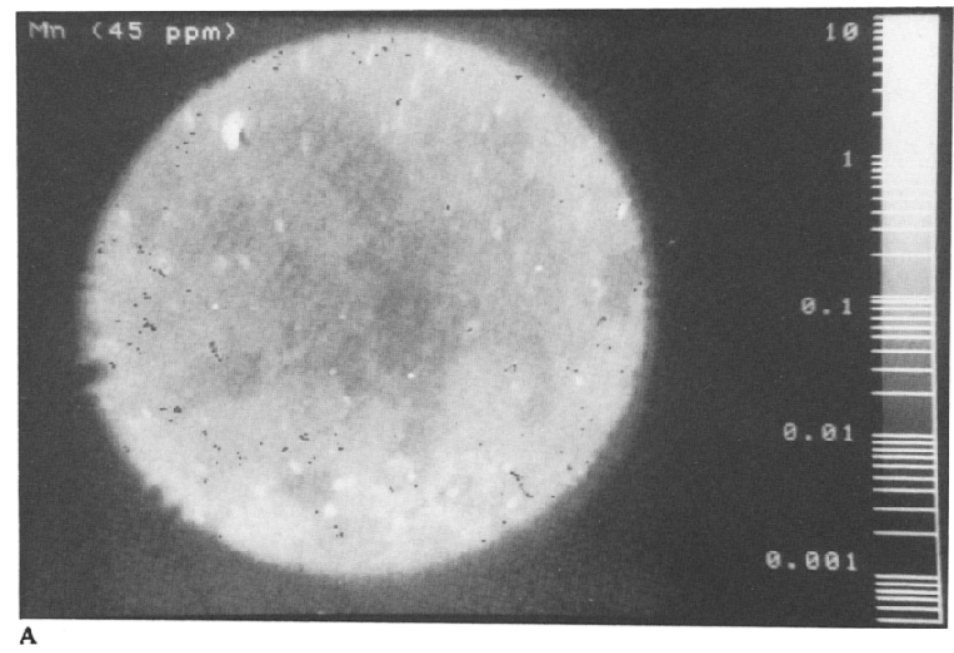

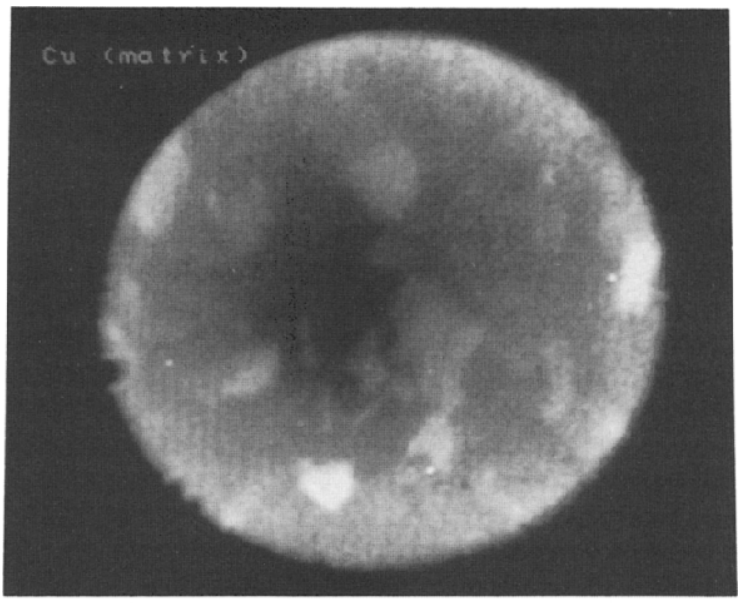

B

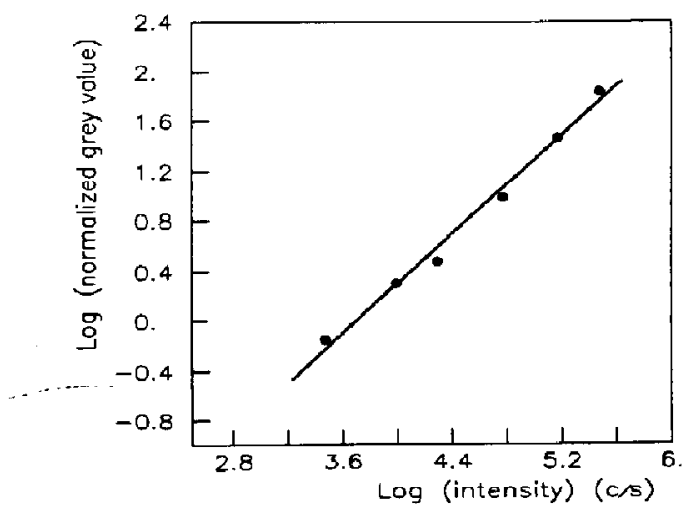

Figure 8. Mean gray value, normalized to the number of integration cycles, versus secondary ion intensity. The number of integration cycles for the different points was 1, 2, 5, 10, 20, and 50 for decreasing secondary ion intensity.
Figure 7. (a) Logarithmic Mn+ image as composed from Figure 6. The scale is in arbitrary units and indicates a dynamic range of more than 3 orders of magnitude. (b) $\mathrm{Cu}^{+}$(matrix) image of the same region. The $\mathrm{Mn}^{+}$and $\mathrm{Cu}^{+}$images are complementary.

one integration cycle, which is, apart from being less noisy, identical to the real-time image. Figure $6 \mathrm{~b}-\mathrm{e}$ corresponds to $3,9,27$, and 81 integration cycles. As explained above, the pixels that had a gray value above $85(255 / 3)$ in Figure 6a will be out of range in Figure $6 \mathrm{~b}$ and may have any value between 0 and 255 . It is clear that as the number of integration cycles increases, information about the very low intensities (e.g., in the middle of the image) becomes available.

From these five images, an image with an increased dynamic range can be composed. Figure 7 a shows the result on a logarithmically scaled image. The dynamic range in this image is now limited by the background of the camera, which is equivalent to about 0.004 count/s per pixel, yielding an overall dynamic range of a factor 2000 . The physical relevance of the intensity variations in the low-intensity region of the logarithmic image is demonstrated by the complementarity to the $\mathrm{Cu}^{+}$matrix ion image in Figure $7 \mathrm{~b}$. The 


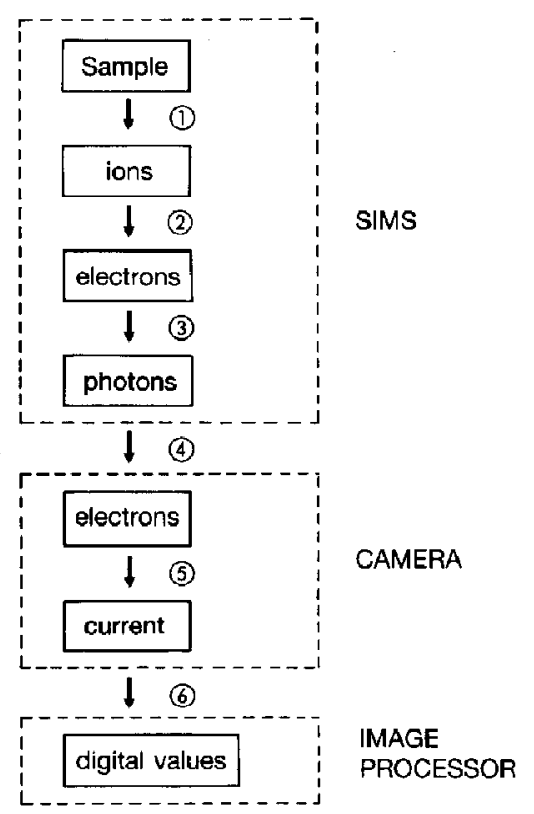

Figure 9. Scheme of all the transformations necessary to convert an elemental distribution into a gray-value image.

differences in intensity in this image are mainly due to crystallographic contrast.

On the other hand, the complementarity does not prove that the intensity ratios within the logarithmic image are not distorted. To evaluate the appropriateness of this approach, the apparatus was tuned to a secondary ion current of $3 \times 10^{5}$ counts/s and the image acquisition parameters were kept constant. The secondary ion current was varied over 2 orders of magnitude, and the number of integration cycles was 1,2 , $5,10,20$, and 50 for the respective points in Figure 8. Within the investigated interval, no deviations from linearity were observed. Experimental practice shows that extended dynamic range imaging (EDRIM) is a very powerful and elegant method.

\section{Quantification of Ion Images}

Figure 9 shows schematically all the transformations involved in the generation of a gray-value image. Table 2 summarizes a number of analytical parameters that are important at the respective stages, together with possible artifacts. The analytical parameters may be externally variable (e.g., the primary bombarding species) or may escape the operators' control (e.g., residual gas adsorption and charging).

The factors that are listed for the first conversion (apart from detection parameters such as electron multiplier efficiency) correspond to the analytical parameters in classical (nonimaging) SIMS. Parameters listed at other stages are related to the image formation and image acquisition components.
Table 2. Summary of a number of analytical parameters and possible artifacts of importance in the conversion of an elemental concentration map into a gray-value image for a camera-based detection system ${ }^{\mathrm{a}}$

\begin{tabular}{ll}
\hline $\begin{array}{l}\text { Conversion } \\
\text { step }\end{array}$ & Analytical parameters and artifacts \\
\hline 1 & Primary ion species \\
& Primary ion energy \\
& Current density \\
& Secondary accelerating voltage \\
& Mass resolutior \\
& Transmission \\
& Contrast and field aperture \\
& Residual gas adsorption \\
& Mass discrimination \\
& Preferential sputtering, knock-on, and \\
& bombardment-induced migration \\
& Crystallographic, topographic, and \\
& energy contrast \\
& Matrix effects \\
& Microchannel plate voltage \\
& Mass cffoct \\
& Energy effect \\
& Blooming \\
& Shading and background \\
& Nonlinearities and saturation \\
Limited quantum efficiency \\
SIT accelerating valtage \\
Camera gain \\
Black level \\
Aberrations \\
Blooming and cross-talk \\
Nonlinearities \\
\hline 5
\end{tabular}

See also Figure 9.

The task of image quantification requires the reconstruction of the sample composition from the measured digital values and can be split into two steps.

1. Reconstruction of the local secondary ion intensity from the obtained digital values. This is the reverse of transformations 2-6 in Figure 9.

2. Conversion of the local secondary ion intensities to local concentrations. This is the reverse of conversion 1 and is largely equivalent to the quantification issue in classical SIMS.

For the reconstruction of the local secondary ion intensity, the influence of all the factors listed in Table 2 must be studied and effectively controlled. This huge task can be avoided if the local gray value is linear with respect to the local secondary ion intensity. If this assumption is valid, the problem of quantification, in terms of local secondary ion intensities, is essentially reduced to equating the integrated gray values to the secondary ion intensity as measured with, for exam- 
ple, the electron multiplier. This implies an important reduction in the experimental workload and a greater ease of utilization, because the parameters in stages 2-6 of Figure 9 do not all have to be taken into account explicitly.

The conversion of an intensity image into a concentration map runs into the classical quantification problems of SIMS and must he tarkled with the commonly used quantification algorithms (e.g., LTE, MISR, or SF). The SF and MISR methods will be studied in this work.

Generally, the relation between the measured grayvalue map $\operatorname{GV}(x, y)$ and the actual element distribution in the area of analysis is given as

$$
\mathrm{GV}(x, y)=A\left(c_{\mathbf{i}}(x, y)\right) B
$$

where the transfer function $A$ describes the conversion of the element distribution [denoted here as the internal concentration contrast $\left.c_{\mathrm{i}}(x, y)\right]$ to secondary ions, and $B$ is the transfer function that describes the conversion of an intensity map to a gray-value image.

Function $A$ can be decomposed into a number of contributions:

$$
A=c_{\mathrm{i}}(x, y) \cdot c_{\mathrm{e}}(x, y) \cdot E \cdot T \cdot \mathrm{C}
$$

where $c_{\mathrm{e}}(x, y)$ is the external concentration contrast, which reflects the matrix effects, and the $E, T$, and $C$ functions describe the influence of energy contrast, topographic contrast, and crystallographic contrast, respectively.

As described above, the microchannel plate and the camera transfer function $B$ can generally be written as

$$
B=\mathrm{MCP} \cdot \mathrm{CF}
$$

where $\mathrm{MCP}$ and $\mathrm{CF}$ denote the transfer function of the microchannel plate and camera, respectively.

Combining eqs 4 and 5 yields

$$
\mathrm{GV}(x, y)=c_{\mathrm{i}}(x, y) c_{\mathrm{e}}(x, y) E \text { TC MCP CF }
$$

and the concentration map $c_{\mathrm{i}}(x, y)$ can be calculated if all the transfer functions are known. In experimental practice, it is not necessary to determine all the individual transfer functions if a reference species is used.

For samples like those used in this study, which are highly conductive, there is no energy contrast in the samples analyzed, and this term (E) can be left out. The topographic and crystallographic contrasts are essentially independent of the ionic species involved. Because these factors are nondiscriminative, they can be omitted in the ratio.

The microchannel plate and camera transfer functions (MCP and $\mathrm{CF}$ ) depend on a great number of parameters. It seems reasonable, however, in view of the analytical characteristics described above, to assume that the factors do not distort the intensity ratios within an image and that the overall microchannel plate and camera transfer functions for the species of interest and the reference species are identical except for a factor $K$ that reflects the difference in amplification. This assumption implies that the shading is independent of the species being imaged and independent of the intensity.

Combining eq 6 for the unknown $(u)$ and the reference (ref) gives

$$
\frac{G V^{\mathrm{u}}(x, y)}{G V^{\mathrm{ref}}(x, y)}=\frac{c_{\mathrm{i}}^{\mathrm{u}}(x, y) c_{\mathrm{e}}^{\mathrm{u}}(x, y)}{c_{\mathrm{i}}^{\text {ref }}(x, y) c_{\mathrm{e}}^{\text {ref }}(x, y)} K
$$

where $c_{\mathrm{e}}^{\mathrm{u}}$ and $c_{\mathrm{e}}^{\text {ref }}$ are the contributions of the external concentration contrast and reflect the matrix effect. They correspond physically to the number of secondary ions emitted per unit concentration. Therefore, the ratio $c_{\mathrm{e}}^{\mathrm{u}} / c_{\mathrm{e}}^{\text {ref }}$ can be replaced by the sensitivity factor $\operatorname{SF}(x, y)$, which can be determined from external standard reference materials.

If we assume that the reference element is homogeneously distributed, the only unknown factor in eq 7 is the constant $K$, which reflects the amplification of the camera-MCP system. $K$ can be determined experimentally by equating the integrated gray-value image $(\Sigma)$ to the total secondary ion current $\left(I_{s}\right)$, as determined with the electron multiplier, for example. The concentration map can then be calculated according to

$$
c_{\mathrm{i}}^{\mathrm{u}}(x, y)=\frac{\mathrm{GV}^{\mathrm{u}}(x, y) \Sigma^{\mathrm{ref}} / I_{\mathrm{s}}^{\mathrm{ref}}}{\mathrm{GV}^{\mathrm{ref}}(x, y) \Sigma^{\mathrm{u}} / I_{s}^{\mathrm{u}}} c_{\mathrm{i}}^{\text {ref }}(x, y) \frac{1}{\mathrm{SF}(x, y)}
$$

The way the factor $\operatorname{SF}(x, y)$ is treated depends on the quantification algorithm selected. In the SF method, the value is equal over the entire area of analysis and is determined from an external standard. In the case of the MISR method, the SF varies locally and is determined from the MISR map.

This quantification procedure was implemented in a FORTRAN program on a microVax. The quantification of an element map takes about half an hour when all the intermediary images are output. It is expected that this computing time can be cut down appreciably by using the image processor for the calculations. For this exploratory study, the microVax was preferred because it provides a virtually unlimited dynamic range and better protection against errors such as overflow and rounding. The dynamic range is especially important because the measured secondary ion currents, and thus the normalized images, may vary over several orders of magnitude, and the dynamic range effectively increases when the ratio of images is taken. Implementation of this procedure on an image processor with a 16-bit-deep processor and 8-bit-deep image memory is, in principle, possible, and its implementation may dramatically increase the speed and flexibility of the procedure. 
Table 3. Main analytical conditions for SIMS

\begin{tabular}{|c|c|c|c|}
\hline & $\begin{array}{c}\text { MISR } \\
\text { calibration }\end{array}$ & $\begin{array}{c}\text { MISR } \\
\text { images }\end{array}$ & SF \\
\hline \multicolumn{4}{|l|}{ Primary } \\
\hline Species & $\mathrm{Ar}^{+}$ & $\mathrm{Ar}^{+}$ & $\mathrm{O}_{2}^{+}$ \\
\hline $\begin{array}{l}\text { Accelerating voltage } \\
(\mathrm{kV})\end{array}$ & 12.5 & 12.5 & 12.5 \\
\hline Intensity (nA) & 780 & 800 & 400 \\
\hline Raster $(\mu \mathrm{m})$ & 500 & 500 & 500 \\
\hline \multicolumn{4}{|l|}{ Secondary } \\
\hline Polarity & + & + & + \\
\hline $\begin{array}{l}\text { Extraneous voltage } \\
\text { (V) }\end{array}$ & 4500 & 4500 & 4500 \\
\hline Imaged field $(\mu \mathrm{m})$ & 400 & 400 & 400 \\
\hline $\begin{array}{l}\text { Contrast diameter } \\
(\mu \mathrm{m})\end{array}$ & 60 & 60 & 60 \\
\hline Field diameter $(\mu \mathrm{m})$ & 1800 & 1800 & 1800 \\
\hline Analyzed area $(\mu \mathrm{m})$ & 400 & 400 & 400 \\
\hline Backfill (Pa) & Variable & $4 \times 10^{-4}$ & $<1 \times 10^{-5}$ \\
\hline Energy slit position $|\mathrm{V}\rangle$ & 0 & 0 & -60 \\
\hline Energy slit width $(V)$ & 130 & 130 & 28 \\
\hline
\end{tabular}

\section{Application to Steel and Aluminum Samples}

To evaluate the described methodology, the experimental and real elemental distributions in a sample have to be compared. No samples are perfectly characterized at the micrometer level, and no reference techniques can yield reliable information in the concentration range from a few percent down to the partsper-million level with an information depth of a few nanometers, so this approach was not possible. Therefore, we compared the mean concentration of the elemental concentration map obtained experimentally with the certified concentration of a number of impurity elements in standard reference materials. We used the SF method and the MISR method for steel and aluminum samples, respectively. The NBS 663 and Aluminium Péchiney (AP) 5042/114 standard reference materials were treated as unknown samples. The NBS 662 and AP 5044/88 were used as external references.

\section{Sample Preparation and Experimental Conditions}

Before analysis, the samples were embedded in tinbismuth eutectic alloy, ground and polished with diamond paste (25, 15, 7, and $1 \mu \mathrm{m}$ ) to avoid topographical effects. The samples were presputtered for about $10 \mathrm{~min}$. The main analytical conditions for SIMS are summarized in Table 3 . For the SF method, oxygen bombardment was used because it increases and stabilizes the secondary ion current. For the MISR method, argon bombardment with oxygen backfill was used.

The number of integration cycles (each consisting of 256 frames) for image acquisition was varied in steps of a factor of 3 , starting from 1 . The maximum number of integration cycles was reached when all parts of the image were sufficiently illuminated. For the steel and aluminum samples, respectively, 11 and 7 impurity elements were recorded, together with the $\mathrm{Fe}^{+}, \mathrm{AlO}^{+}$, and $\mathrm{Al}_{2}^{+}$ion images. The isotopic composition of the impurity elements was verified to detect possible interferences.

\section{Sensitivity Factor Method on Steel Samples}

For the SF method, the reference SFs were determined on three different spots to minimize the influence of heterogeneity, and the $\mathrm{Fe}^{+}$intensity was used as a reference species. To obtain the concentration images, the normalized images of the element of interest were multiplied by the SF map, which is homogeneous, because the $\mathrm{SF}$ is assumed to have the same value over the entire area of analysis. Figures $10-13$ show a few examples. Figure 10 shows the $\mathrm{Fe}^{+}$intensity map in counts per second per pixel. Owing to shading effects, the intensity in the center of the analyzed area is only $50 \%$ of the intensity at the outside. At the outer edge, the intensity is cut away by the field diaphragm.

Figure 11 shows the concentration map of vanadium. The shading, which was present in the intensity map, is greatly reduced in the concentration map. Vanadium is homogeneously distributed at a level of about $0.25 \%$ and shows a number of inclusions, where the concentration may rise to about $1 \%$. The certified mean concentration of the sample is $0.31 \%$, which agrees with the concentration map.

Figure 12 shows the power of the EDRIM method. The concentration map of titanium is displayed on a logarithmic scale, covering the entire concentration range from $50 \mathrm{ppm}$ to $5 \%$. The inclusions have concentrations up to several percent, whereas the homogeneous background signal is of the order of $100 \mathrm{ppm}$ or below. The zirconium and niobium concentration maps (not shown here) also display the presence of intense inclusions. The cross-talk of the very intense $\mathrm{Zr}$ spots is clearly visible on the logarithmic images. The presence of the spots diminishes the intensity of the pixels to the right of the spot. If the spots are extremely intense, there may even be cross-talk to the beginning of the next line (because the frame is read out in lines from left to right and from top to bottom). The $\mathrm{Zr}$ and $\mathrm{Nb}$ are exclusively present in a number of inclusions, which may have a concentration of up to a few percent. The concentration of the homogeneously distributed fraction cannot be determined exactly because the intensity is close to the detection limit. Only an upper limit of $300 \mathrm{ppm}$ can be stated. The $\mathrm{Zr}$ and $\mathrm{Nb}$ inclusions are at the same location, indicating that the elements are present in the same inclusions. Inclusions with a higher $\mathrm{Zr}$ content appear to have a lower $\mathrm{Nb}$ content and vice versa.

In some cases, the shading effect is not successfully corrected by normalizing to the matrix ion intensity. Figure $13 a$ and $b$ shows molybdenum intensity and 

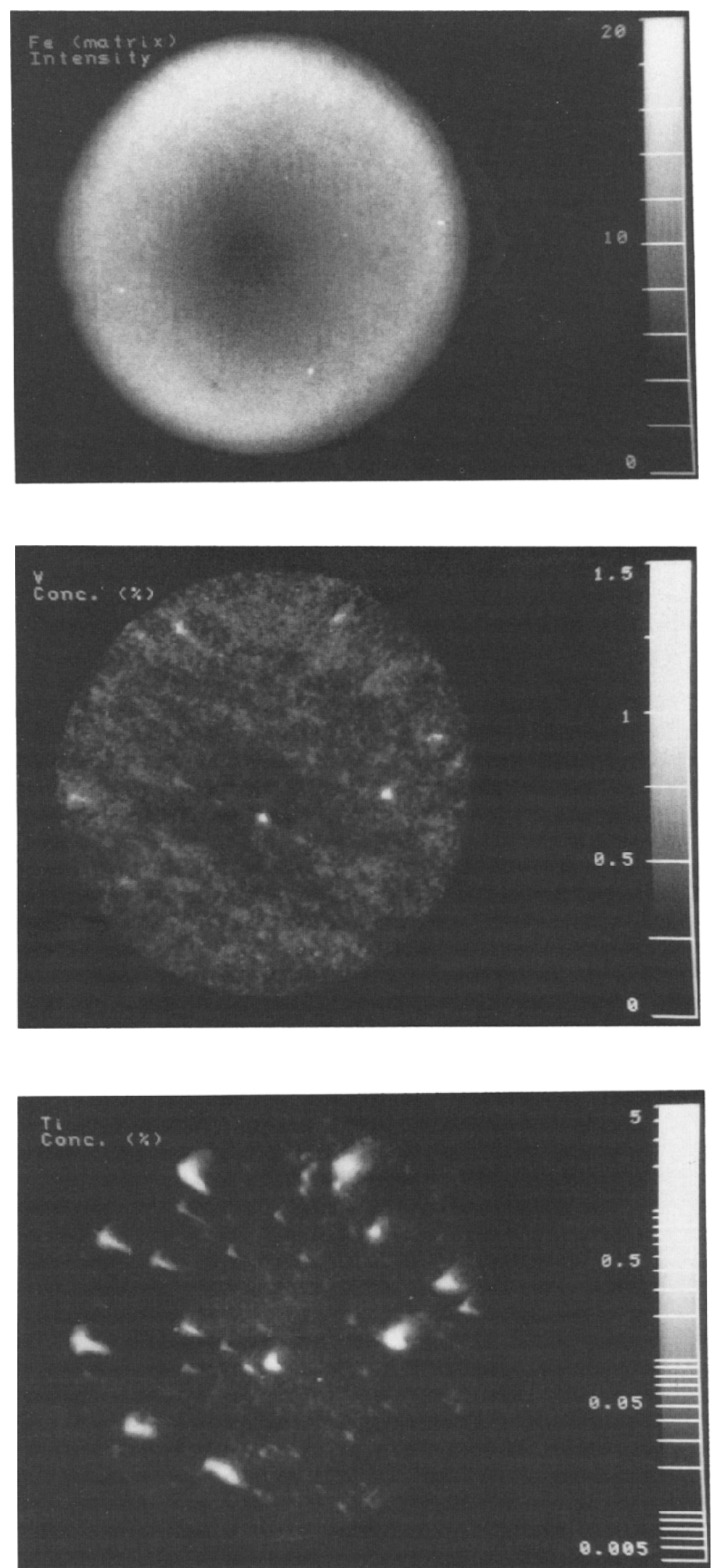

Figure 10. $\mathrm{Fe}^{+}$intensily map of the NBS 663 steel sample. The shading in the image is clearly observable.

Figure 11. Concentration map of vanadium in the NBS 663 steel standard obtained with the SF method.
Figure 12. Logarithmic concentration map of titanium in the NBS 663 steel sample. With extended dynamic range imaging, the concentration map covers the entire concentration range from $50 \mathrm{ppm}$ to $5 \%$. 


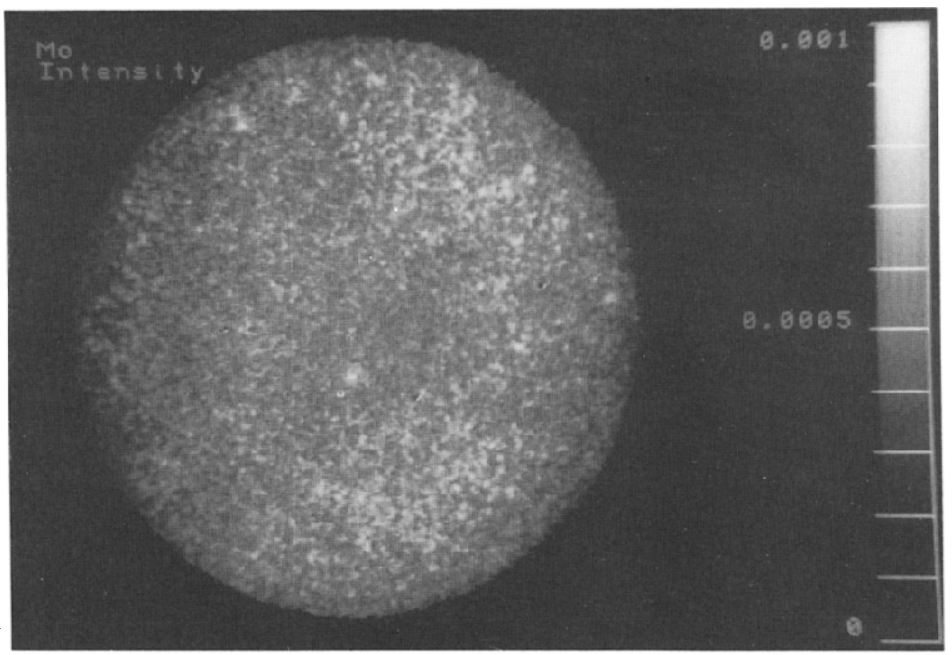

A

Figure 13. (a) Intensity and (b) concentration maps of molybdenum in the NBS 663 steel sample. There is no shading in the intensity image, and the concentration map shows inverse shading.

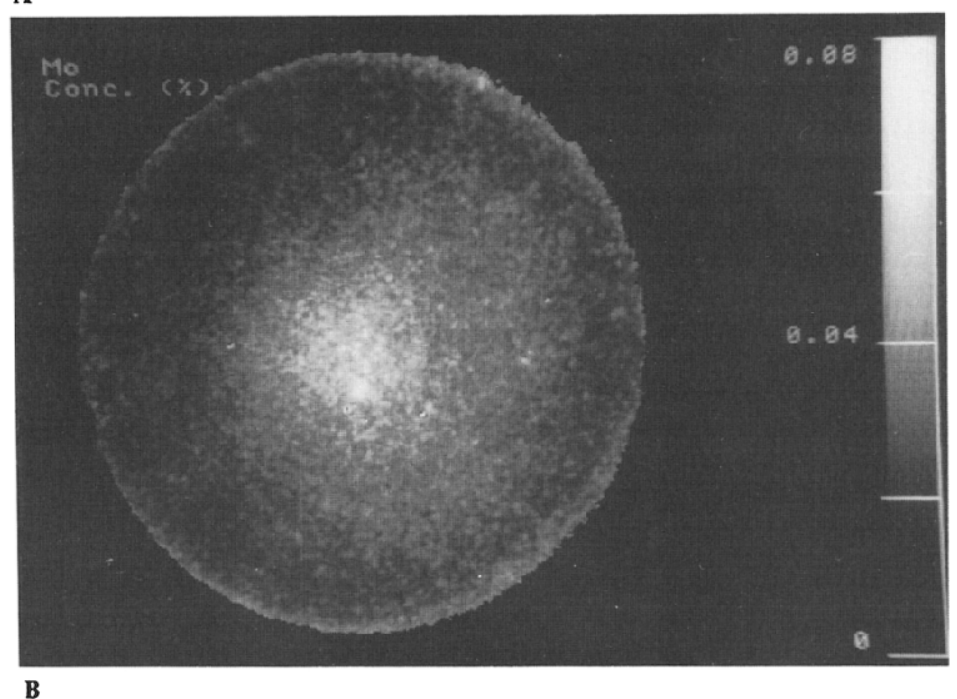

concentration maps. The Mo intensity map shows no shading, and as a consequence the "corrected" concentration map shows "inverse shading." Boron also shows this behavior. The certified mean concentration of B is 9 ppm, which demonstrates the sensitivity of the technique.

For these images, the normalization to the matrix ion image appears not to be a good way to overcome the shading problem, which is attributed to the way the images are generated. For high secondary ion currents, a high-quality image that does not vary with time is obtained. The camera can easily be adjusted to obtain a well-scaled image with maximum gray values within scale. In the case of very low secondary ion currents, for example, 10 counts/s, there is no visible image. The individual secondary ions induce flashes distributed over the area of analysis. To detect all the ions, the camera is operated at a high amplification, and the bursts of light are swept out of scale. This means that the shading effect is absent, because all flashes, both in the middle of the screen and at the outside, have a momentary gray value of 255 .

This hypothesis is supported by the observation that it is mainly the elements with a very low secondary ion intensity that show this absence of shading. Table 4 summarizes the experimental observations. Zirconium, niobium, and titanium are not included in the table, because they are present only in inclusions and there is no reliable information with respect to the presence or absence of shading. The elements are ordered in descending ion intensity of the homogeneously distributed fraction of the element. The shading before and after correction is listed and is denoted by a minus sign for a decrease in the middle of the area of analysis, a zero if no shading is present, and a plus sign for inverse shading, which means an increase in the center.

Table 4 clearly illustrates that the extent of shading is related to the intensity. An intensity of 0.001 count/s per pixel is roughly the turning point. Below this inten- 
Table 4. Shading before and after normalization to the $\mathrm{Fe}^{+}$reference image as a function of the intensity of the homogeneous fraction in the image

\begin{tabular}{lccc}
\hline Element & $\begin{array}{c}\text { Intensity of the } \\
\text { homogeneous fraction } \\
\text { [counts/s' pixell }\end{array}$ & $\begin{array}{c}\text { Shading } \\
\text { before } \\
\text { correction }\end{array}$ & $\begin{array}{c}\text { Shading } \\
\text { after } \\
\text { correction" }\end{array}$ \\
\hline $\mathrm{B}$ & 0.0002 & 0 & + \\
$\mathrm{Mo}$ & 0.0006 & 0 & + \\
$\mathrm{Cu}$ & 0.0008 & 0 & + \\
$\mathrm{Ni}$ & 0.001 & $1-1$ & $1+1$ \\
$\mathrm{Si}$ & 0.07 & - & 0 \\
$\mathrm{~V}$ & 0.2 & - & 0 \\
$\mathrm{Mn}$ & 0.4 & - & 0 \\
$\mathrm{Cr}$ & 0.4 & - & 0 \\
$\mathrm{Fe}$ & 10 & - & 0 \\
\hline
\end{tabular}

* - intensity smaller in center of image; 0 , no shading; + , inverse shading (intensity greater in center of image).

sity, there is no shading in the original images; above it, a shading correction becomes necessary.

The elements can be classified according to their distribution. A number of elements such as $\mathrm{B}, \mathrm{Ni}$, Mo, and $\mathrm{Cr}$ are homogeneously distributed, whereas $\mathrm{Zr}$, $\mathrm{Nb}$, and $\mathrm{Ti}$ are present almost exclusively in inclusions. The elements $\mathrm{Cu}, \mathrm{V}$, and $\mathrm{Mn}$ show an intermediate behavior; they are present both in inclusions and in a relatively important homogeneous fraction. Moreover, there is a striking similarity between the element maps of the heterogeneously distributed elements, suggesting the existence of inclusions that contain precipitated materials of a large number of elements. This phenomenon was also observed in other matrices [28].

Table 5 compares the mean concentration in the maps with the certified concentrations. The contribution of heterogeneity was estimated from the triple measurement on the reference sample. The mean relative deviation between the certified and experimental

Table 5. Comparison of the certified and experimental concentrations of eleven impurity elements in the NBS 663 steel sample, as determined with the SF method

\begin{tabular}{lrll}
\hline & & \multicolumn{2}{c}{ Concentration (wt \%) } \\
\cline { 3 - 4 } Element & $\begin{array}{r}\text { Heterogeneitya } \\
(\%)\end{array}$ & Certified & Experimental \\
\hline Mn & 6 & 1.50 & 1.56 \\
$\mathrm{Si}$ & 60 & 0.74 & 0.38 \\
$\mathrm{Cu}$ & 14 & 0.098 & 0.15 \\
$\mathrm{Ni}$ & 9 & 0.32 & 0.33 \\
$\mathrm{Cr}$ & 9 & 1.31 & 1.61 \\
$\mathrm{~V}$ & 11 & 0.31 & 0.39 \\
$\mathrm{Mo}$ & 5 & 0.030 & 0.027 \\
$\mathrm{Nb}$ & 4 & 0.049 & 0.045 \\
$\mathrm{~B}$ & 3 & 0.00091 & 0.00067 \\
$\mathrm{Zr}$ & 16 & 0.049 & 0.047 \\
$\mathrm{Ti}$ & 7 & 0.050 & 0.040 \\
\hline
\end{tabular}

- The contribution of heterogeneity was estimated from a triple analysis on the reference sample. cuncentrations is $22 \%$. This is a good agreement, especially since the estimated contribution of heterogeneity is $13 \%$. For silicon, the larger deviation is probably due to heterogeneity.

\section{Matrix Ion Species Ratio Method for Aluminum Samples}

The MISR curves of seven elements were determined from the external standard AP 5044/88, and $\mathrm{AlO}^{+} / \mathrm{Al}_{2}^{+}$ was chosen as the matrix ion species ratio. Figure 14 gives the MISR curves of the elements. For calculational purposes, the experimental data were fitted with the linear functions indicated on the figure. The oxygen backfill pressure for the analysis of the unknown

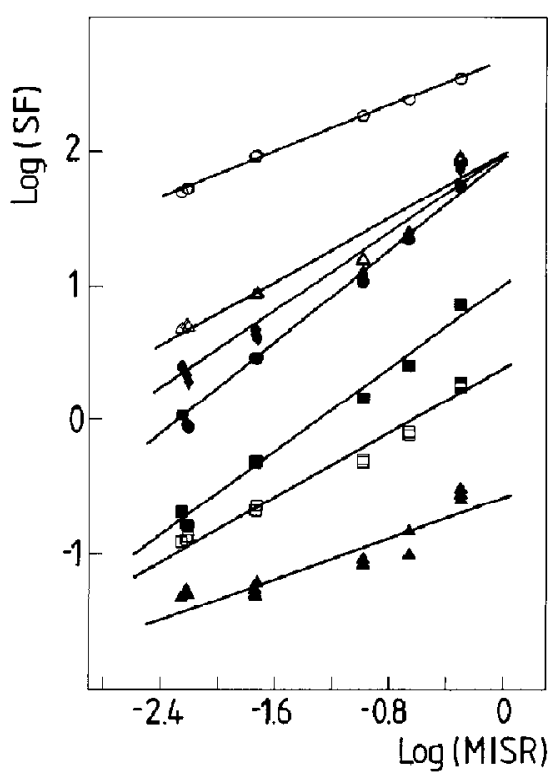

Figure 14. MISR curves of $\mathrm{Mg}(O), \mathrm{Ti}(\Delta), \mathrm{Mn}(\bullet), \mathrm{Fe}(\bullet), \mathrm{Cu}$ (G), Si $(\square)$, and $\mathrm{Pb}(\boldsymbol{\Lambda})$ in aluminum. 
Figure 15. $\mathrm{AlO}^{+} / \mathrm{Al}_{2}^{+}$MISR image of the aluminum sample.

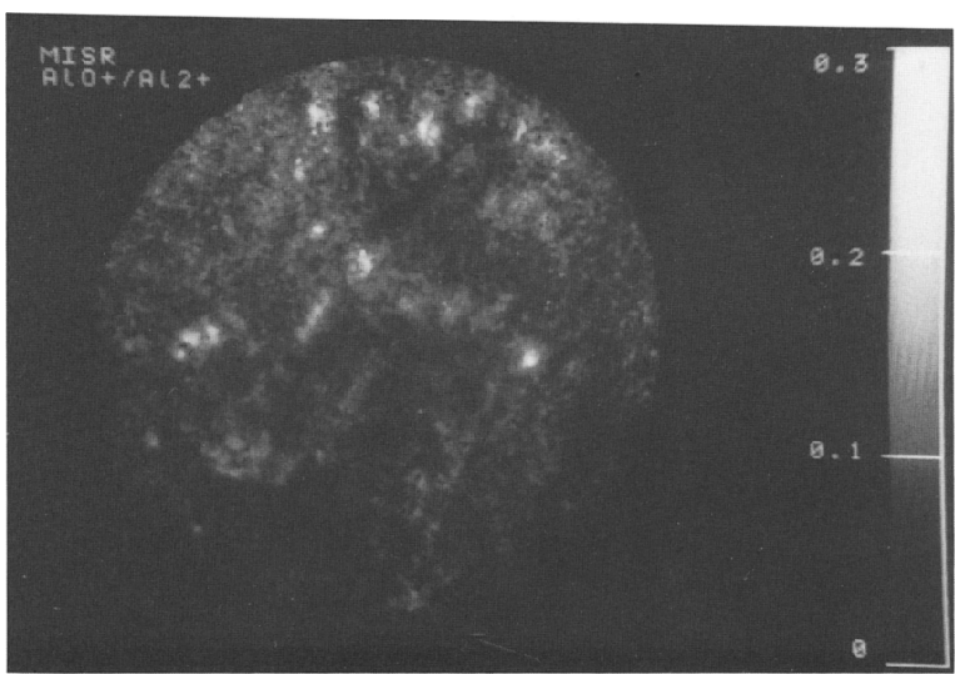

sample was adjusted to obtain an overall MISR value of 0.05 . This allows correction for both positive and negative deviations of the local MISR value without extrapolating the experimental MISR curves.

Figure 15 shows the $\mathrm{AlO}^{+} / \mathrm{Al}_{2}^{+} \mathrm{MISR}$ map. The MISR values range from 0.03 to 0.3 and are well within the investigated experimental range, ensuring valid SF values. There is a spread of a factor of 10 within the image, suggesting that there will be important changes in the local sensitivity of the elements.

Figure 16a-c illustrates the calculation of a concentration map. The extended dynamic range image of $\mathrm{Fe}^{+}(\mathrm{a})$ is divided by the $\mathrm{Al}_{2}^{+}$reference image, yielding a normalized $\mathrm{Fe}^{+}$image. From the data of the MISR curve and the MISR map, the SF image of Fe is calculated (b). Assuming a constant concentration of the reference element over the area of analysis, the concentration map can be calculated from the SF map and the normalized intensity map. Figure $16 \mathrm{c}$ shows the concentration map. The Fe concentration varies from 0.04 to $2 \%$. The certified concentration is $0.35 \%$. The SF map shows a variation in the sensitivity of Fe of the order of a factor 5 .

Table 6 compares the experimental and certified concentrations. The experimental concentrations are determined in two ways. For column 2, the mean of the concentration map was calculated, whereas for column 3 the calculation was performed from the ion currents, treating the whole image at once. The results are very similar and agree well with the certified concentrations. The overall relative error is $16 \%$.

\section{Evaluation of the Results}

The relative error between the experimental and certified concentrations is $22 \%$ for the SF method and $16 \%$ for the MISR method. This is a good agreement and indicates that the developed methodology is success- ful, although it does not actually prove that the values obtained for local concentrations are accurate.

The shading correction as applied here is not suitable for very low secondary ion currents. This problem can be solved by recording the shading planes for different secondary ion currents or by leaving out a shading correction for images that give no evidence of diminished sensitivity in the center of the area of analysis. This shading effect is usually readily separable from real intensity variations in the image because of its typical manifestation as a dip in the center of the image. It can therefore also be corrected for by means of image processing, for example, by dividing by a lowpass filtered image.

Although the SF and MISR methods both provide good results, there is an important difference in their sensitivity to local variations in the matrix composition. If the results from previous studies [20] can be extrapolated to these matrices, it is clear that the MISR method is far more sensitive to local variations in the matrix composition. For a number of elements, the concentration of the impurity elements in the inclusions may be of the order of a few percent, and the local matrix composition does not agree with the mean composition of the sample. This local matrix effect will probably cause less severe problems for the SF method, because it is less susceptible to local differences in matrix composition if an appropriate reference species is selected. The MISR method usually yields more reproducible results and is better able to correct for local differences in oxygen content.

In this respect, SIMS is to be considered a technique that is complementary to other surface analysis techniques such as Auger electron spectroscopy (AES). If the local concentration of the impurities is very important, the matrix effect prevents accurate determinations with SIMS, but other, less sensitive techniques are available. If the local concentration of the impu- 


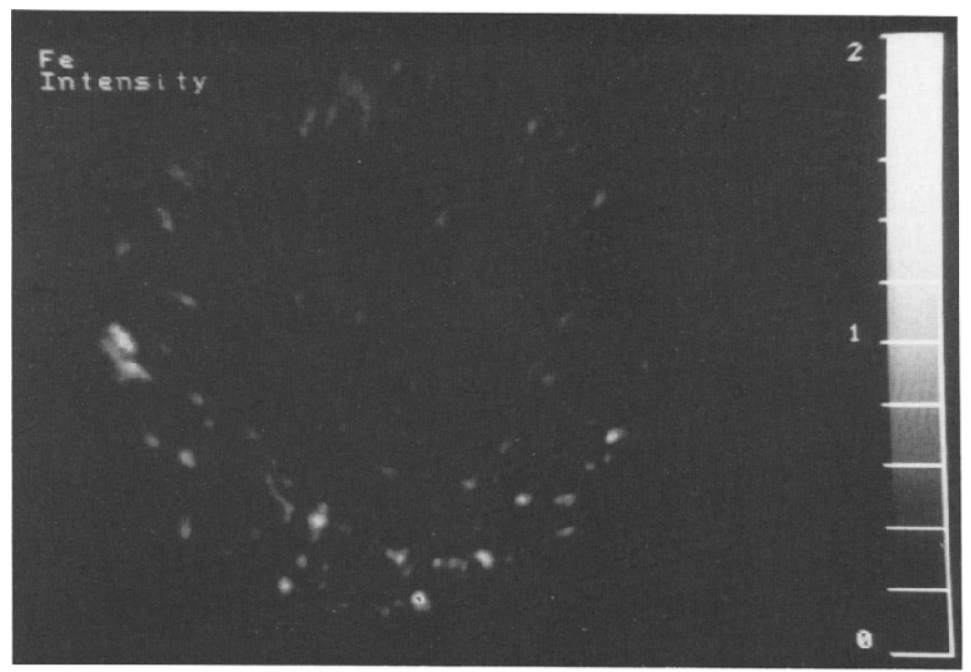

A

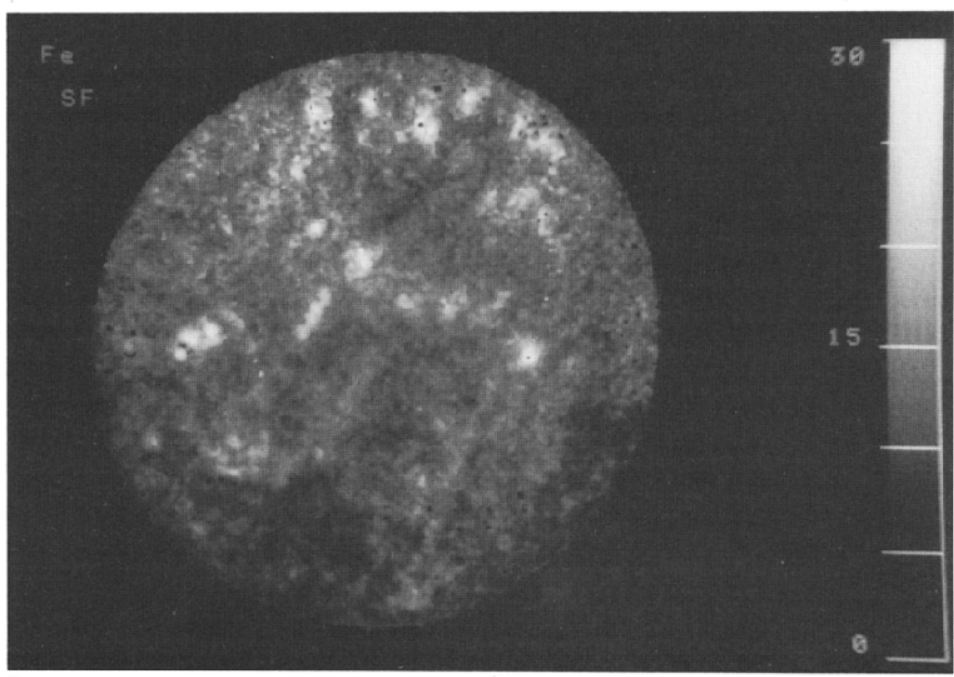

B

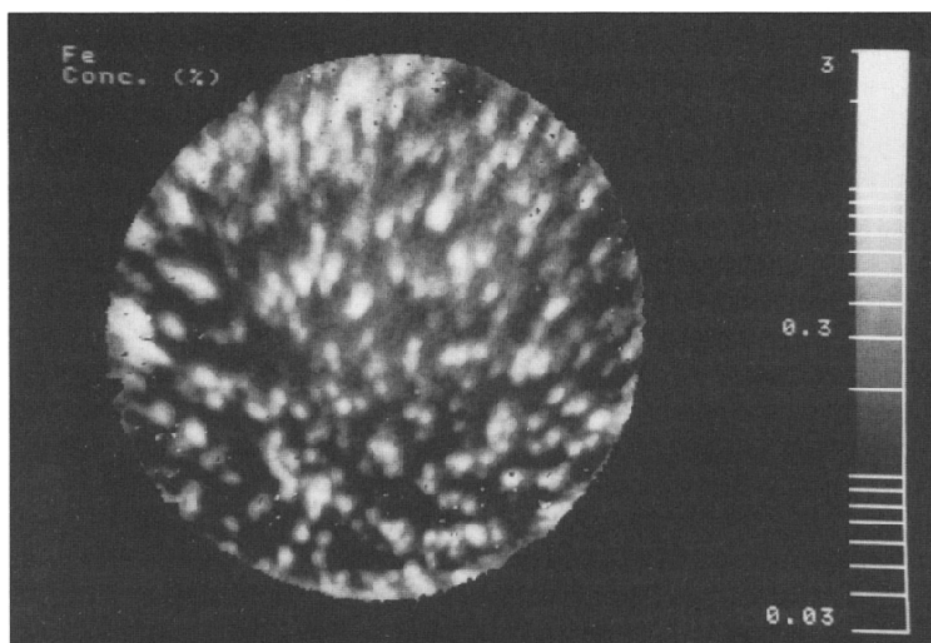

Figure 16. Example showing the calculation of the Fe concentration image with the MISR method. (a) The intensity image. (b) SF map calculated from the MISR map. (c) A concentration image can be calculated from the normalized intensity image and the SF map. 
Table 6. Comparison of certified and experimental concentrations of seven impurity elements in the AP 5042/114 aluminum reference sample, as obtained with the MISR method

\begin{tabular}{llll}
\hline & \multicolumn{3}{c}{ Concentration (wt \%) } \\
\cline { 2 - 4 } & & \multicolumn{2}{c}{ Experimental } \\
\cline { 3 - 4 } Element & Certified & Per pixel & Total image \\
\hline $\mathrm{Mg}$ & 0.15 & 0.14 & 0.15 \\
$\mathrm{Ti}$ & 0.0030 & 0.0052 & 0.0050 \\
$\mathrm{Mn}$ & 0.31 & 0.30 & 0.31 \\
$\mathrm{Fe}$ & 0.35 & 0.31 & 0.31 \\
$\mathrm{Cu}$ & 7.10 & 5.7 & 5.9 \\
$\mathrm{Si}$ & 0.30 & 0.29 & 0.28 \\
$\mathrm{~Pb}$ & 0.35 & 0.35 & 0.35 \\
\hline
\end{tabular}

rities is very low, SIMS does not suffer from matrix effects to the same extent.

\section{Conclusion}

The implementation of a high-sensitivity camera is a convenient way to extend the detection sensitivity of the Cameca IMS-3f down to the level of individual secondary ions. In combination with state-of-theart image processing equipment, it provides a flexible and powerful tool for ion microscopy. The quantitative characteristics of the camera and the microchannel plate-camera system were evaluated. It was shown that the use of multiple exposures can extend the dynamic range within one image up to three orders of magnitude without losing quantitative information. The problem of image quantification was described and tested for two common SIMS quantification methods, the SF method and the MISR method. The shading correction originally applied in this study proved to be unsuitable for low-intensity images. The cause of this problem and remedies to overcome it were assessed. The comparison of the experimental and certified mean concentrations in the sample show that the method is successful, although they do nut actually prove that the values obtained for local concentrations are valid. In the inclusions, in particular, where the local matrix composition may differ appreciably from the overall matrix composition, results are subject to error. In this respect, SIMS can be considered complementary to less sensitive surface analysis techniques that can be used to determine the high levels of impurities in the inclusions.

\section{Acknowledgments}

We are indebted to Dr. Jacob from the medicine department. F. Michiels and P. Van Espen are indebted to the Belgium National Science Fund (N.F.W.O.) for financial support. This work was carried out under grant no. 4.0002.90.

\section{References}

1. Castaing, R.; Slodzian, G. J. Microsc. 1962, 1, 395.

2. Schilling, J. H.; Buger, P. A. Int. J. Mass. Spectrom. Ion Phys. $1978,27,283$.

3. Steiger, W.; Ruedenauer, F. G. Anal. Chem. 1979, 51, 2107.

4. Fasset, J. D.; Drummer, D. M.; Morrison, G. H. Anal. Chim. Acta 1979, 112, 165.

5. Levi-Setti, R.; Wang, Y. L.; Crow, B. J. Phys. 1984, 45, C9-C197.

6. Bayly, A. R.; Waugh, A. R.; Vohralik, P. Spectrochim. Acta 1985, 40B, 717.

7. Bernius, M. T.; Ling, Y. C.; Morrison, G. H. In Secondary Ion Mass Spectrometry; Benninghoven, A.; Huber, A. M.; Werner, H. W.; Eds.; Wiley: New York, 1988; Vol. 6, p 377.

8. Odom, R. W.; Furman, B. K.; Evans, C. A.; Bryson, C. E.; Peterson, W. A.; Kelly, M. A.; Wayne, D. H. Anal. Chem. 1983, 55, 574.

9. Furman, B. K.; Morrison, G. H. Anal. Chem. 1980, 52, 2305.

10. Bryan, S. R.; Woodward, W. S.; Linton, R. W.; Griffis, D. P. J. Vac. Sci. Technol. A 1985, 3, 2102.

11. Thorne, N. A.; Dubus, A.; Degrève, F. Scanning Electron Microsc. 1986, 4, 1255.

12. Leta, D. P. In Secondary Ion Mass Spectrometry, Benninghoven, A.; Colton, R. J.; Simons, D. S.; Werner, H. W.; Eds.; Springer Verlag: New York, 1986; Vol. 5, p 232.

13. Odom, R. W.; Wayne, D. H.; Evans, C. A., Jr. In Secondary Ion Mass Spectrometry, Benninghoven; A. Ohano, J.; Shimizu, R;; Werner, H. W.; Eds.; Springer Verlag: New York, 1984; Vol. 4, p 186.

14. Ruedenauer, F. G. Surface Interface Anal. 1984, 6(3), 132.

15. Kingham, D. R.; Bayly, A. R.; Fathers, D. J.; Vohralik, P.; Walls, J. M.; Waugh, A. R. Scanning Microsc. 1987, 1, 463.

16. Andersen, C. A.; Hinthorne, J. R. Anal. Chem. 1973, 45, 1421.

17. Morgan, A. E.; Werner, H. W. J. Chem. Phys. 1978, 68, 3900.

18. Morgan, A. E.; Werner, H. W. Anal. Chem. 1977, 49, 927.

19. Ganjei, J.; Leta, D.; Morrison, G. H. Anal. Chem. 1978. 50, 285.

20. Michiels, F.; Adams, F. J. Anal. At. Spectrom. 1987, 2, 773.

21. Leta, D. P.; Morrison, G. H. Anal. Chem. 1980, 52, 277.

22. Fasset, J. D.; Roth, J. R.; Morrison, G. H. Anal. Chem. 1977, 49, 2322.

23. Drummer, D. M.; Morrison, G. H. Anal. Chem. 1980, 52, 2147.

24. Patkin, A. J.; Chandra, S.; Morrison, G. H. Anal. Chem. 1982, $54,2507$.

25. Patkin, A. J.; Morrison, G. H. Anal. Chem. 1982, 54, 2.

26. Radermacher, L.; Schilling, J. H.; Beske, H. E. Fresenius Z Anal. Chem. 1984, 319, 724

27. Schilling, J. H.; Radermacher, L.; Beske, H. E. Fresenitus Z. Anal. Chem. 1984, 319, 622.

28. Liu, X. D.; Michiels, F, Van Espen, P.; Adams, F. Mikrochim. Acta 1986, 3, 49. 